\title{
Neuroimaging Biomarkers of mTOR Inhibition on Vascular and Metabolic Functions in Aging Brain and Alzheimer's Disease
}

\begin{abstract}
Jennifer Lee ${ }^{1}$, Lucille M. Yanckello1,2, David Ma', Jared D. Hoffman 1,2, Ishita Parikh', Scott Thalman ${ }^{3}$, Bjoern Bauer ${ }^{4}$, Anika M. S. Hartz ${ }^{1,2}$, Fahmeed Hyder ${ }^{5}$ and Ai-Ling Lin ${ }^{1,2,3 *}$

'Sanders-Brown Center on Aging, University of Kentucky, Lexington, KY, United States, ${ }^{2}$ Department of Pharmacology and Nutritional Science, University of Kentucky, Lexington, KY, United States, ${ }^{3}$ F. Joseph Halcomb III, M.D. Department of Biomedical Engineering, University of Kentucky, Lexington, KY, United States, ${ }^{4}$ Department of Pharmaceutical Sciences, University of Kentucky, Lexington, KY, United States, ${ }^{5}$ Departments of Radiology and Biomedical Engineering, Magnetic Resonance Research Center, Yale University, New Haven, CT, United States
\end{abstract}

The mechanistic target of rapamycin (mTOR) is a nutrient sensor of eukaryotic cells. Inhibition of mechanistic mTOR signaling can increase life and health span in various species via interventions that include rapamycin and caloric restriction (CR). In the central nervous system, mTOR inhibition demonstrates neuroprotective patterns in aging and Alzheimer's disease (AD) by preserving mitochondrial function and reducing amyloid beta retention. However, the effects of mTOR inhibition for in vivo brain physiology remain largely unknown. Here, we review recent findings of in vivo metabolic and vascular measures using non-invasive, multimodal neuroimaging methods in rodent models for brain aging and AD. Specifically, we focus on pharmacological treatment (e.g., rapamycin) for restoring brain functions in animals modeling human AD; nutritional interventions (e.g., CR and ketogenic diet) for enhancing brain vascular and metabolic functions in rodents at young age (5-6 months of age) and preserving those functions in aging (18-20 months of age). Various magnetic resonance (MR) methods [i.e., imaging $(\mathrm{MRI})$, angiography (MRA), and spectroscopy (MRS)], confocal microscopic imaging, and positron emission tomography (PET) provided in vivo metabolic and vascular measures. We also discuss the translational potential of mTOR interventions. Since PET and various MR neuroimaging methods, as well as the different interventions (e.g., rapamycin, $\mathrm{CR}$, and ketogenic diet) are also available for humans, these findings may have tremendous implications in future clinical trials of neurological disorders in aging populations.

Received: 30 April 2018 Accepted: 02 July 2018 Published: 26 July 2018

Citation:

Lee J, Yanckello LM, Ma D Hoffman JD, Parikh I, Thalman S, Bauer B, Hartz AMS, Hyder F and

Lin A-L (2018) Neuroimaging Biomarkers of mTOR Inhibition on Vascular and Metabolic Functions in Aging Brain and Alzheimer's Disease.

Front. Aging Neurosci. 10:225. doi: 10.3389/fnagi.2018.00225
Keywords: mechanistic target of rapamycin (mTOR), rapamycin, caloric restriction, ketogentic diet, MRI, PET,

\section{INTRODUCTION}

The mechanistic target of rapamycin (mTOR) is a nutrient sensor that mediates the responses to energy status and growth factor in eukaryotic cells (Laplante and Sabatini, 2009). Discovered by three groups in 1994, mTOR is a particular protein bound by rapamycin (Brown et al., 1994; Cafferkey et al., 1994; Sabatini et al., 1994). mTOR activity can be inhibited by both rapamycin and Aging, Alzheimer's disease 
nutritional signaling, such as caloric restriction (CR) (Perluigi et al., 2015). Inhibition of mTOR can switch cellular response from reproduction/growth to somatic maintenance, with decreased protein synthesis and cell growth, and increased autophagy in animal models (Harrison et al., 2009; Stanfel et al., 2009). As such, mTOR inhibition has shown to increase resistance to stresses resulting in lifespan extension in various mammalian species, and being considered central to the regulation of both aging and age-related diseases (Johnson et al., 2013).

In the central nervous system, mTOR inhibition has been shown to prevent neurodegeneration and protect brain functions in aging. Notably, rapamycin reduces amyloid-beta $(\mathrm{A} \beta)$ plaques and neurofibrillary tau tangles and improves cognitive functions in mice that model human Alzheimer's disease (AD) (Spilman et al., 2010; Majumder et al., 2011). Similarly, CR (without malnutrition) is able to alleviate $\mathrm{AD}$-like pathology (Lee et al., 2000, 2002; Thrasivoulou et al., 2006). In addition, CR protects mitochondrial function (the powerhouse in the cells), maintains glucose homeostasis, and reduces oxidative stress all phenotypes of aging (Park et al., 2005; Duan and Ross, 2010; Perluigi et al., 2015). Thus, CR (reduced caloric and glucose intake) shifts metabolism toward ketone body utilization (Guo et al., 2015; Lin et al., 2015). Elevated ketone body metabolism or the administration of the ketogenic diet (KD) is also evident to be neuroprotective against $\mathrm{AD}$, aging, epilepsy, brain injury, and neurodegeneration (Van der Auwera et al., 2005; Yang et al., 2017). However, biochemical and molecular experiments may limit mTOR-related research to in vitro or ex vivo cell culture or animal models. Such findings may be incapable of being completely translated and applied to humans.

Powerful brain imaging tools have been refined to visualize changes in brain function in vivo over time (Lavina, 2016; Hyder and Rothman, 2017). In particular, functional imaging can be used to determine changes in physiology before ADlike pathology appears and before the onset of cognitive impairment. Brain vascular and metabolic dysfunction plays a critical role in driving neurodegeneration and dementia (Reiman et al., 2001, 2004, 2005; Thambisetty et al., 2010; Fleisher et al., 2013). We have recently demonstrated that early detection of these physiological changes and identification of effective interventions using imaging would be critical to potentially slow down brain aging and prevent AD. Table 1 summarizes the imaging techniques used in the studies we review, ranging from various magnetic resonance imaging (MRI) and spectroscopy (MRS) methods to positron emission tomography (PET) to confocal microscopic imaging, where the last method is primarily for preclinical research. To assess vascular functions, we used MRI-based arterial spin labeling (ASL), which measures quantitative cerebral blood flow (CBF) values by utilizing arterial blood water as an endogenous tracer. We also determined vascular density with magnetic resonance angiography (MRA) and blood-brain barrier (BBB) P-glycoprotein transport activity with live-cell imaging confocal microscopy. To assess metabolic functions, we used wellestablished PET protocols and proton magnetic resonance spectroscopy $\left({ }^{1} \mathrm{H}\right.$-MRS) to quantify glucose uptake and brain metabolites, respectively (Lin et al., 2012; Lin and Rothman, 2014). We have also included the novel MRS techniques of ${ }^{1} \mathrm{H}\left[{ }^{13} \mathrm{C}\right]$ proton-observed-carbon-edited (POCE) to determine neurotransmission rate and mitochondrial oxidative metabolism in the aging brain.

In this review, we will discuss our neuroimaging findings on mTOR inhibition in the aging and AD brain. First, we will address the effectiveness of rapamycin in reducing AD-like pathology by restoring cerebrovascular functions in mice. Second, we will address our recent findings on $\mathrm{CR}$ and $\mathrm{KD}$ in enhancing brain vascular functions and shifting metabolism in young healthy mice. Third, we will provide evidence that CR preserves brain metabolic and vascular functions in aging in both mice and rats. Finally, we will discuss the translational potential of mTORrelated interventions in future human studies.

\section{RAPAMYCIN RESTORES BRAIN VASCULAR AND METABOLIC FUNCTIONS IN MICE MODELING HUMAN ALZHEIMER'S DISEASE}

Rapamycin was discovered in 1970s from soil samples in Easter Island Rapa Nui (Sehgal et al., 1975); thus, the compound was named rapamycin (also known as sirolimus) after its place of origin (Johnson et al., 2013). It was discovered in 1988 that rapamycin contained immunosuppressive properties (Camardo, 2003). This finding led to the FDA's approval of rapamycin in 1999 as an immunosuppressant preventative of the rejection in organs transplant patients (Camardo, 2003). Over the past two decades, rapamycin or its analogs have been widely used in the clinic and their toxicity profiles have been well characterized (Soefje et al., 2011).

Preclinical studies have been conducted to analyze the potential effectiveness of rapamycin to treat $\mathrm{AD}$ (Caccamo et al., 2010; Spilman et al., 2010; Majumder et al., 2011). In a recent study (Lin et al., 2017a), we focused on the effects of rapamycin in presymptomatic mice carrying the human apolipoprotein 84 (APOE4) allele, given that APOE4 is the most significant genetic risk factor for AD (Liu et al., 2013). Neuroimaging studies in humans have shown that cognitively normal APOE4 carriers develop vascular and metabolic deficits decades before the aggregation of $\mathrm{A} \beta$ and tau tangles (Reiman et al., 2001, 2004, 2005; Thambisetty et al., 2010; Fleisher et al., 2013). In particular, researchers conducting PET studies found that cognitively normal carriers of the APOE4 allele have abnormally low cerebral metabolic rates of glucose (CMRglc) in similar brain regions as patients diagnosed with $\mathrm{AD}$ (Reiman et al., 2001, 2004, 2005; Thambisetty et al., 2010; Fleisher et al., 2013). This metabolic abnormality was observed both in late-middle-aged (40-60 years of age) and young (2039 years of age) carriers, who have normal memory and cognitive ability and are without $A \beta$ or tau pathology. These PET findings suggest that APOE4 carriers develop functional brain abnormalities several decades prior to the potential onset of dementia. Longitudinal research using MRI has displayed 
TABLE 1 | List of discussed neuroimaging methods.

\begin{tabular}{|c|c|c|c|}
\hline Modality & Methods & Measurements & Applications \\
\hline \multirow{4}{*}{$\begin{array}{l}\text { Magnetic resonance imaging (MRI) and } \\
\text { spectroscopy (MRS) }\end{array}$} & ASL & Cerebral blood flow & Humans and animals \\
\hline & MRA & Vascular density & \\
\hline & ${ }^{1} \mathrm{H}-\mathrm{MRS}$ & A variety of essential brain metabolites & \\
\hline & POCE & $\begin{array}{l}\text { Mitochondrial function; Neurotransmission rate; neuronal and } \\
\text { glial activities }\end{array}$ & \\
\hline Positron emission tomography (PET) & ${ }^{18} \mathrm{FDG}$ & Cerebral glucose metabolism & \\
\hline Confocal Microscopy & Live-cell imaging & Blood brain barrier P-gp transport activity & Mainly in animals \\
\hline
\end{tabular}

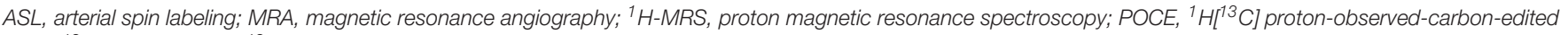
MRS; ${ }^{18}$ FDG, fluorine-18 ( $\left.{ }^{18} \mathrm{~F}\right)$-labeled 2-fluoro-2-deoxy-d-glucose.

that $\mathrm{CBF}$ is reduced in an accelerated manner in similar brain regions (e.g., frontal, parietal, and temporal cortices) in cognitively healthy APOE4 carriers (Thambisetty et al., 2010). The APOE4-related neurovascular risk is strongly correlated with an accelerated decline in verbal memory, language capability, attention, and visual/spatial abilities in midlife (Bangen et al., 2013).

A similar situation is seen in transgenic mice that express the human APOE4 isoform that is driven by the human glial fibrillary acidic protein promoter. Young, presymptomatic APOE4 mice have significantly lower CMRglc and CBF, as well as increased BBB leakage compared to the wild-type (WT) non-APOE4 mice (Bell et al., 2012; Lin et al., 2017a). Treating asymptomatic female APOE4 mice with rapamycin for 1 month resulted in a significant increase in $\mathrm{CBF}$ compared to the non-treated littermates. After 6 months of treatment, we found that rapamycin-treated APOE4 mice had normal CBF that was comparable to that of the sexand age-matched WT mice. Similarly, rapamycin-treated mice also had lower BBB leakage. Furthermore, we found that BBB leakage could potentially be blocked by inhibiting cyclophilin A-dependent proinflammatory pathways with rapamycin (Bell et al., 2012). In addition, CMRglc was also restored to WT level as observed in the rapamycin-treated APOE4 mice (Lin et al., 2017a).

In another study with hAPP(J20) mice (a mouse model of human $\mathrm{AD}$ ) that already developed significant $\mathrm{A} \beta$ pathology and cognitive decline, we found that rapamycin was also effective in restoring neurovascular function. Symptomatic hAPP(J20) 11 month old mice treated with rapamycin for 16 weeks had restored CBF to the level of WT mice (Lin et al., 2013). In addition, rapamycin restored vascular density, determined by MR angiography, in the brains of hAPP(J20) mice. The restored vascular integrity was highly correlated with reduced $\mathrm{A} \beta$, cerebral amyloid angiopathy (CAA), and microhemorrhages in treated hAPP(J20) mice. These findings were consistent with the literature showing that rapamycin can reduce $A \beta$ (Liu et al., 2017). In this study, we also identified that mTOR inhibition activates endothelial nitric oxide synthase (eNOS), and thus, released nitric oxide (NO), a vasodilator (Cheng et al., 2008; Lin et al., 2013). Therefore, rapamycin activating eNOS may be critical for restoring CBF in hAPP(J20) mice. In addition to restored cerebrovascular function and reduced AD-like pathology, hAPP(J20) mice also had improved memory and learning performance after 16 weeks of rapamycin treatment (Lin et al., 2013). Collectively, data generated from the two imaging studies show that rapamycin can potentially prevent $\mathrm{AD}$ phenotypes in APOE4 mice and reverse the effects of AD in hAPP(J20) transgenic mice (Richardson et al., 2015).

\section{CALORIC RESTRICTION AND KETOGENIC DIET ENHANCE BRAIN VASCULAR FUNCTIONS AND SHIFT METABOLISM IN YOUNG MICE}

In the early 1930s, Clive McCay demonstrated that restricting calorie intake without malnutrition can prolong both the mean and maximal lifespan in rats when compared to animals on ad libitum diet (AL; free eating) (McCay et al., 1989; Park, 2010). Since then, CR is perhaps the most studied anti-aging manipulation within a broad range of species (Colman et al., 2009; Choi et al., 2011; Rahat et al., 2011). This is further supported by other studies that display lower incidences of agerelated neurodegenerative disorders found in animals treated with CR (Park et al., 2005; Duan and Ross, 2010).

Recently, our efforts have been focused on understanding how CR impacts brain function in the early stage. In particular, we would like to know how brain vascular and metabolic functions might be impacted with $\mathrm{CR}$ in young mice. We imaged mice at 5-6 months of age, either on $40 \% \mathrm{CR}$ diet or AL, and found that CR significantly enhanced CBF (>20\%) both globally and in the hippocampus, compared to their AL littermates (Parikh et al., 2016). The increase in CBF was associated with reduced $\mathrm{mTOR}$ and increased eNOS levels that were similar to what we observed with rapamycin. In addition, CR-fed mice had significantly increased P-glycoprotein (P-gp) transport activity levels at the $\mathrm{BBB}$, which facilitates clearance of $\mathrm{A} \beta$ out of the brain. These findings are consistent with literature showing that $\mathrm{CR}$ reduces $\mathrm{AD}$-like pathology and the onset of cognitive impairment (Mouton et al., 2009; Schafer et al., 2015).

We used ${ }^{1} \mathrm{H}$-MRS to determine energy metabolites in the hippocampus (Guo et al., 2015). We observed that CR mice displayed significantly increased levels of total creatine ( $\mathrm{tCr}$ ), 
a high-energy substrate, in comparison to AL mice. Given that $\mathrm{tCr}$ is the sum of creatine and phosphocreatine, we posited that $\mathrm{CR}$ increases adenosine triphosphate (ATP) production in young CR mice since phosphocreatine acts in a central role as an intracellular buffer during ATP production in mitochondria. This finding is consistent with literature that CR enhances ATP production by activating AMP-activated protein kinase (AMPK) and sirtuins pathways, which in turn suppresses the mTOR pathway (Blagosklonny, 2010). It has been found that the level of glucose regulates the AMPK pathway. With low levels of glucose and metabolic stress that accompany CR, there is a depletion of energy (low ATP: AMP ratio), which in turn activates AMPK (Salt et al., 1998; Hardie, 2014). AMPK, when activated, can be seen as an indicator of cellular energy status, turning on catabolic pathways that generate ATP while inhibiting cellular processes that consume ATP such as the mTOR pathway.

We also found significantly elevated levels of taurine in the CR mice when compared to the AL mice. Since taurine is correlated with neuromodulation, higher levels imply that young CR mice might have augmented excitability compared to the age-matched AL mice. Interestingly, both globally and in the hippocampus and frontal cortex (regions related to cognitive functions), CR mice displayed significantly reduced brain glucose uptake as determined by PET imaging (Guo et al., 2015). Our imaging findings are consistent with Western blot data showing that $\mathrm{CR}$ reduces glucose transporter 1 (GLUT-1) in brain capillaries of the mice (Parikh et al., 2016). As a result, we found a mismatch of CBF-CMRglc coupling induced by $\mathrm{CR}$, opposite to tight coupling of CBFCMRglc in a normal brain at rest (Fox et al., 1988; Lin et al., 2010).

These reduced glucose uptake results led us to hypothesize that in order to sustain essential mitochondrial activity and neuronal functions, the brain may utilize alternative fuel substrates as an energy source. As the brain would also use ketone bodies as energy source (Akram, 2013), we measured ketone body levels in the brain and blood and found that CR rodents had a significantly higher concentration of ketone bodies in comparison to AL animals (Guo et al., 2015; Lin et al., 2015). The findings indicated that CR may, at a very early stage in the brain, induce a metabolic switch from glucose to ketones.

To verify the impact of elevated ketone bodies on vascular functions, we fed young, age-matched WT mice with the KD for 16 weeks. Similar to what we observed with young $\mathrm{CR}$ mice, mice fed with $\mathrm{KD}$ also had significant increases in CBF and P-gp transport activity levels in brain capillaries compared to control mice (Ma et al., 2018). These neurovascular enhancements were also associated with reduced mTOR and increased eNOS protein expressions. The result is consistent with previous reports that ketogenesis is associated with the down-regulation of mTOR (McDaniel et al., 2011). In line with this, two other studies indicate that an acute increase in ketone body concentration (via infusion of $\beta$-hydroxyl butyrate) elevated $\mathrm{CBF}$ independent of overall cerebral metabolic activity. This suggests that ketone bodies can directly increase CBF via the cerebral endothelium (Hasselbalch et al., 1996; Roy et al., 2013).

\section{CALORIC RESTRICTION PRESERVES BRAIN VASCULAR AND METABOLIC FUNCTIONS IN AGING RODENTS}

Healthy aging is accompanied by $\mathrm{CBF}$ reduction, $\mathrm{BBB}$ impairment and $A \beta$ retention (Lin et al., 2015; Parikh et al., 2016; Hoffman et al., 2017). To identify CR effects on the aging brain, we included old CR and AL mice (18-20 months of age) in the same CR experiments and compared them with young mice (5-6 months of age). We found that CR enhanced $\mathrm{CBF}$ in young mice; moreover, $\mathrm{CR}$ also reduced the CBF decline in aging (Parikh et al., 2016). As a result, when compared to young $\mathrm{AL}$ mice, old $\mathrm{CR}$ mice had comparable levels of $\mathrm{CBF}$, indicating that $\mathrm{CR}$ preserves $\mathrm{CBF}$ with age. Similar results were found in rats, showing that old rats with chronic CR diet had much higher CBF compared to the agematched animal, and had comparable CBF level compared to the young AL rats (Lin et al., 2015). These results support that $\mathrm{CR}$ has an early enhancement effect on CBF that is preserved with aging. In addition, the preserved $\mathrm{CBF}$ in the hippocampus and frontal cortex were highly associated with the preserved memory and learning, as well as the reduced anxiety (Parikh et al., 2016). Our results suggest that dietary intervention initiated at a young age (e.g., young adults) may prove beneficial in the preservation of cognitive and mental abilities in aging.

A similar trend was also observed in hippocampal $\mathrm{tCr}$ concentration. As mentioned above, $\mathrm{tCr}$ was enhanced in young $\mathrm{CR}$ mice. Although $\mathrm{tCr}$ dropped dramatically as the $\mathrm{CR}$ mice getting older, $\mathrm{tCr}$ levels remained comparable to those in young $\mathrm{AL}$ mice and were higher than those in old $\mathrm{AL}$ mice. This suggests that CR increases ATP production in young CR mice while preserving ATP production in old CR mice (Guo et al., 2015).

Using advanced MRS techniques like POCE, we were able to trace in vivo mitochondrial oxidative metabolisms in neurons and neurotransmitter cycling between neuronal and glial cells (Lin et al., 2014). We found that, compared with the young AL rats, old CR rats had similar levels of neuronal glucose oxidation and neurotransmitter cycling, suggesting CR preserved mitochondrial functions and neuronal activity with age. In contrast, old AL rats had much lower levels in both measures. When calculating the ATP production rates for the three groups we found that old $\mathrm{CR}$ and young $\mathrm{AL}$ animals also had comparable levels of ATP production. We also observed metabolic shifts in aging animals. When compared to age-matched AL rats, old CR rats had significantly lower glucose uptake values in the various brain regions but had significantly higher levels of ketone bodies, $\beta$-hydroxyl butyrate (BHB) in the brain (Lin et al., 2015). The metabolic shift may play a critical role in sustaining brain energetics in aging. 
Taken together, using multi-modal imaging methods we demonstrated that CR enhances vascular and metabolic functions in early life stages and decelerates the decline with age. Maintaining a healthy brain homeostasis may be due to the metabolic shift from glucose to ketone bodies (Lin et al., 2017b).

\section{TRANSLATIONAL POTENTIAL OF MTOR INTERVENTIONS IN CLINICAL TRIALS}

Many mTOR inhibitors (including rapamycin, rapalogs, and Everolimus) have already been approved by the FDA and are widely used in clinics (Soefje et al., 2011). Since 1999, rapamycin, alongside other immunosuppressive agents, has been administered to transplant patients to prevent the rejection of organs (Camardo, 2003). Over the past decade, studies also showed that rapamycin or rapalogs have an anti-tumor property; for relatively long periods of time, cancer patients with rapamycin show little change in their quality of life (Mita et al., 2003). Other studies reported that Everolimus improved cognition and reduced depression in humans (Lang et al., 2009). Recent studies showed that with low doses of rapamycin (e.g., lower than half of the therapeutic dosage; $0.5 \mathrm{mg}$ daily or $5 \mathrm{mg}$ weekly), cognitively normal elderly had improved immune functions with minimal side effects (Mannick et al., 2014). The results of the studies support that a short-term rapamycin treatment can be used safely in otherwise healthy older person.

To date, most rapamycin and rapalog clinical studies focus on structural neuroimaging to examine changes in brain tumor mass (Tillema et al., 2012; Fukumura et al., 2015; Ma et al., 2015; Sasongko et al., 2016), metastatic cancer (Subbiah et al., 2015), or active lesions (Moraal et al., 2010). However, functional neuroimaging such as EEG has been clinically applied to assess the efficacy of rapamycin in treating epilepsy (Cambiaghi et al., 2015), and MRS was used to study metabolic implications of rapamycin (Serkova et al., 1999).

CR has also been studied in humans. A recent publication by Redman et al. shows that young, healthy individuals having achieved 15\% CR experienced about $8 \mathrm{~kg}$ weight loss over 2 years (Redman et al., 2018). Energy expenditure (measured over $24 \mathrm{~h}$ of awake and sleep cycle) was reduced beyond weight loss and systemic oxidative stress was also reduced. Findings from this 2 year CR trial in healthy, non-obese humans provide new evidence of persistent systemic metabolic slowing accompanied by reduced oxidative stress, which supports the rate of living and oxidative damage theories of mammalian aging.

CR has also been observed to improve memory in older adults (Fontan-Lozano et al., 2008; Witte et al., 2009; Mattson, 2010; Valdez et al., 2010). Using functional and structural MRI measurements, Witte et al. (2014) reported that resveratrol, a CR-mimetic nutrient, enhanced word retention over a 30min period in older adults when compared with placebo.

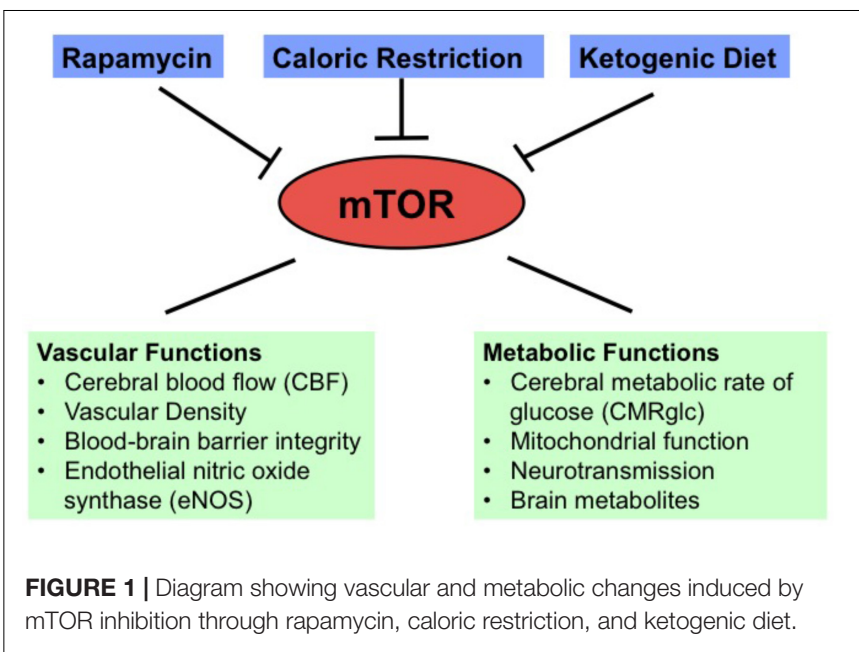

These results support that supplementary resveratrol can improve memory performance, as well as improve glucose metabolism and increase hippocampal functional connectivity in older adults. In another study, Jakobsdottir et al. (2016) reported that $\mathrm{CR}$ reserved abnormal brain activity in brain areas (e.g., amygdala) involved in the processing of visual food-related stimuli in postmenopausal women with obesity. It should be noted, however, there are studies that these studies have only investigated the short-term benefits of CR.

The KD has been used in the clinic to treat epilepsy (Baranano and Hartman, 2008; Walczyk and Wick, 2017), Parkinson's disease (Vanitallie et al., 2005), and autism (Evangeliou et al., 2003). The use of neuroimaging in clinical KD studies include EEG and functional MRI to define the extent of dysplasia (Guerrini et al., 2015), and ${ }^{1} \mathrm{H}-\mathrm{MRS}$ to assess GABAergic activity (Wang et al., 2003) and glucose metabolism (Fujii et al., 2007). Recent studies also investigated the efficacy of ketone utilization in the brain. Using PET, it was found that the cerebral metabolic rate of ketones represents about 33\% of the brain's energy requirements after 4 days on KD (Courchesne-Loyer et al., 2017). POCE studies in human have reported that consumption of ketones (BHB) is predominantly neuronal (Pan et al., 2002). These results support that ketone bodies are an effective alternative fuel substrate in the non-fasted adult human brain.

Collectively, rapamycin, $\mathrm{CR}$, and $\mathrm{KD}$ have been widely applied to human studies, which indicates that our work with animal models has the potential to be translated to human studies. To date, little has been reported regarding in vivo vascular and metabolic measures in aging and $\mathrm{AD}$ with these interventions. The use of quantitative neuroimaging methods (e.g., ${ }^{18}$ FDG-PET, POCE, ${ }^{1}$ H-MRS, MRA, and ASL) would be vital in future use to identify the efficacy of mTOR-related interventions and treatments for protecting brain functions in aging and various $\mathrm{AD}$-related neurodegeneration, including vascular dementia and Down syndrome (Lin et al., 2016). 


\section{CONCLUSION}

In this review, we discussed the neuroprotective effects of mTOR inhibition in aging and AD. Specifically, rapamycin is a preventative, and possibly a treatment, for the effects of the AD phenotype observed in APOE4 and hAPP(J20) transgenic mouse models of $\mathrm{AD}$; $\mathrm{CR}$ and $\mathrm{KD}$ can enhance brain vascular functions and shift metabolism in young healthy mice; and CR can preserve brain metabolic and vascular functions in aging. We summarize these findings in Figure 1. As the quantitative PET and MRI neuroimaging methods used in these studies in animal models can be translated into human studies, they will be greatly useful in future studies to examine the effects of these mTOR-related interventions in preventing brain function declines associated with aging and neurodegeneration in clinical trials.

\section{REFERENCES}

Akram, M. (2013). A focused review of the role of ketone bodies in health and disease. J. Med. Food 16, 965-967. doi: 10.1089/jmf.2012.2592

Bangen, K. J., Beiser, A., Delano-Wood, L., Nation, D. A., Lamar, M., Libon, D. J., et al. (2013). APOE genotype modifies the relationship between midlife vascular risk factors and later cognitive decline. J. Stroke Cerebrovasc. Dis. 22, 1361-1369. doi: 10.1016/j.jstrokecerebrovasdis.2013.03.013

Baranano, K. W., and Hartman, A. L. (2008). The ketogenic diet: uses in epilepsy and other neurologic illnesses. Curr. Treat Options Neurol. 10, 410-419. doi: 10.1007/s11940-008-0043-8

Bell, R. D., Winkler, E. A., Singh, I., Sagare, A. P., Deane, R., Wu, Z., et al. (2012) Apolipoprotein E controls cerebrovascular integrity via cyclophilin A. Nature 485, 512-516. doi: 10.1038/nature 11087

Blagosklonny, M. V. (2010). Calorie restriction: decelerating mTOR-driven aging from cells to organisms (including humans). Cell Cycle 9, 683-688. doi: 10.4161/ cc.9.4.10766

Brown, E. J., Albers, M. W., Shin, T. B., Ichikawa, K., Keith, C. T., Lane, W. S., et al. (1994). A mammalian protein targeted by G1-arresting rapamycin-receptor complex. Nature 369, 756-758. doi: 10.1038/369756a0

Caccamo, A., Majumder, S., Richardson, A., Strong, R., and Oddo, S. (2010) Molecular interplay between mammalian target of rapamycin (mTOR), amyloid-beta, and Tau: effects on cognitive impairments. J. Biol. Chem. 285, 13107-13120. doi: 10.1074/jbc.M110.100420

Cafferkey, R., Mclaughlin, M. M., Young, P. R., Johnson, R. K., and Livi, G. P. (1994). Yeast TOR (DRR) proteins: amino-acid sequence alignment and identification of structural motifs. Gene 141, 133-136. doi: 10.1016/03781119(94)90141-4

Camardo, J. (2003). The Rapamune era of immunosuppression 2003: the journey from the laboratory to clinical transplantation. Transplant. Proc. 35(Suppl. 3), 18S-24S. doi: 10.1016/S0041-1345(03)00356-7

Cambiaghi, M., Magri, L., and Cursi, M. (2015). Importance of EEG in validating the chronic effects of drugs: suggestions from animal models of epilepsy treated with rapamycin. Seizure 27, 30-39. doi: 10.1016/j.seizure.2015.02.015

Cheng, C., Tempel, D., Oostlander, A., Helderman, F., Gijsen, F., Wentzel, J., et al. (2008). Rapamycin modulates the eNOS vs. shear stress relationship. Cardiovasc. Res. 78, 123-129. doi: 10.1093/cvr/cvm103

Choi, J. S., Choi, K. M., and Lee, C. K. (2011). Caloric restriction improves efficiency and capacity of the mitochondrial electron transport chain in Saccharomyces cerevisiae. Biochem. Biophys. Res. Commun. 409, 308-314. doi: 10.1016/j.bbrc.2011.05.008

Colman, R. J., Anderson, R. M., Johnson, S. C., Kastman, E. K., Kosmatka, K. J., Beasley, T. M., et al. (2009). Caloric restriction delays disease onset and mortality in rhesus monkeys. Science 325, 201-204. doi: 10.1126/science. 1173635

Courchesne-Loyer, A., Croteau, E., Castellano, C. A., St-Pierre, V., Hennebelle, M., and Cunnane, S. C. (2017). Inverse relationship between brain glucose and

\section{AUTHOR CONTRIBUTIONS}

All authors listed have made a substantial, direct and intellectual contribution to the work, and approved it for publication.

\section{FUNDING}

The studies were supported by National Institutes of Health (NIH)/National Institute on Aging (NIA) Grant K01AG040164, NIH/NIA Grant R01AG054459, NIH/CTSA Grant UL1TR000117, and American Federation for Aging Research Grant \#A12474 to A-LL, NIH/NIA Grant R01AG039621 to AH, NIH/NINDS Grant R01NS079507 to BB, NIH/NIMH Grant R01MH067528 to FH, and NIH Training Grant T32DK007778 to JH, and T32AG057461 to ST.

ketone metabolism in adults during short-term moderate dietary ketosis: a dual tracer quantitative positron emission tomography study. J. Cereb. Blood Flow Metab. 37, 2485-2493. doi: 10.1177/0271678X16669366

Duan, W., and Ross, C. A. (2010). Potential therapeutic targets for neurodegenerative diseases: lessons learned from calorie restriction. Curr. Drug Targets 11, 1281-1292. doi: 10.2174/1389450111007011281

Evangeliou, A., Vlachonikolis, I., Mihailidou, H., Spilioti, M., Skarpalezou, A., Makaronas, N., et al. (2003). Application of a ketogenic diet in children with autistic behavior: pilot study. J. Child Neurol. 18, 113-118. doi: 10.1177/ 08830738030180020501

Fleisher, A. S., Chen, K., Liu, X., Ayutyanont, N., Roontiva, A., Thiyyagura, P., et al. (2013). Apolipoprotein E epsilon4 and age effects on florbetapir positron emission tomography in healthy aging and Alzheimer disease. Neurobiol. Aging 34, 1-12. doi: 10.1016/j.neurobiolaging.2012.04.017

Fontan-Lozano, A., Lopez-Lluch, G., Delgado-Garcia, J. M., Navas, P., and Carrion, A. M. (2008). Molecular bases of caloric restriction regulation of neuronal synaptic plasticity. Mol. Neurobiol. 38, 167-177. doi: 10.1007/s12035-0088040-1

Fox, P. T., Raichle, M. E., Mintun, M. A., and Dence, C. (1988). Nonoxidative glucose consumption during focal physiologic neural activity. Science 241, 462-464. doi: 10.1126/science.3260686

Fujii, T., Ho, Y. Y., Wang, D., De Vivo, D. C., Miyajima, T., Wong, H. Y., et al. (2007). Three Japanese patients with glucose transporter type 1 deficiency syndrome. Brain Dev. 29, 92-97. doi: 10.1016/j.braindev.2006.07.007

Fukumura, S., Watanabe, T., Takayama, R., Minagawa, K., and Tsutsumi, H. (2015). Everolimus treatment for an early infantile subependymal giant cell astrocytoma with tuberous sclerosis complex. J. Child Neurol. 30, 1192-1195. doi: $10.1177 / 0883073814544703$

Guerrini, R., Duchowny, M., Jayakar, P., Krsek, P., Kahane, P., Tassi, L., et al. (2015). Diagnostic methods and treatment options for focal cortical dysplasia. Epilepsia 56, 1669-1686. doi: 10.1111/epi.13200

Guo, J., Bakshi, V., and Lin, A. L. (2015). Early shifts of brain metabolism by caloric restriction preserve white matter integrity and long-term memory in aging mice. Front. Aging Neurosci. 7:213. doi: 10.3389/fnagi.2015. 00213

Hardie, D. G. (2014). AMP-activated protein kinase: maintaining energy homeostasis at the cellular and whole-body levels. Annu. Rev. Nutr. 34, 31-55. doi: 10.1146/annurev-nutr-071812-161148

Harrison, D. E., Strong, R., Sharp, Z. D., Nelson, J. F., Astle, C. M., Flurkey, K., et al. (2009). Rapamycin fed late in life extends lifespan in genetically heterogeneous mice. Nature 460, 392-395. doi: 10.1038/nature08221

Hasselbalch, S. G., Madsen, P. L., Hageman, L. P., Olsen, K. S., Justesen, N., Holm, S., et al. (1996). Changes in cerebral blood flow and carbohydrate metabolism during acute hyperketonemia. Am. J. Physiol. 270, E746-E751. doi: 10.1152/ajpendo.1996.270.5.E746

Hoffman, J. D., Parikh, I., Green, S. J., Chlipala, G., Mohney, R. P., Keaton, M., et al. (2017). Age drives distortion of brainmetabolic, vascular and cognitive 
functions, and the gut microbiome. Front. Aging Neurosci. 9:298. doi: 10.3389/ fnagi.2017.00298

Hyder, F., and Rothman, D. L. (2017). Advances in imaging brain metabolism. Annu. Rev. Biomed. Eng. 19, 485-515. doi: 10.1146/annurev-bioeng-071516044450

Jakobsdottir, S., Van Nieuwpoort, I. C., Van Bunderen, C. C., De Ruiter, M. B., Twisk, J. W., Deijen, J. B., et al. (2016). Acute and short-term effects of caloric restriction on metabolic profile and brain activation in obese, postmenopausal women. Int. J. Obes. 40, 1671-1678. doi: 10.1038/ijo.2016.103

Johnson, S. C., Rabinovitch, P. S., and Kaeberlein, M. (2013). mTOR is a key modulator of ageing and age-related disease. Nature 493, 338-345. doi: 10.1038/ nature 11861

Lang, U. E., Heger, J., Willbring, M., Domula, M., Matschke, K., and Tugtekin, S. M. (2009). Immunosuppression using the mammalian target of rapamycin (mTOR) inhibitor everolimus: pilot study shows significant cognitive and affective improvement. Transplant. Proc. 41, 4285-4288. doi: 10.1016/j. transproceed.2009.08.050

Laplante, M., and Sabatini, D. M. (2009). mTOR signaling at a glance. J. Cell Sci. 122, 3589-3594. doi: 10.1242/jcs.051011

Lavina, B. (2016). Brain vascular imaging techniques. Int. J. Mol. Sci. 18:E70. doi: 10.3390/ijms 18010070

Lee, J., Duan, W., Long, J. M., Ingram, D. K., and Mattson, M. P. (2000). Dietary restriction increases the number of newly generated neural cells, and induces BDNF expression, in the dentate gyrus of rats. J. Mol. Neurosci. 15, 99-108. doi: 10.1385/JMN:15:2:99

Lee, J., Seroogy, K. B., and Mattson, M. P. (2002). Dietary restriction enhances neurotrophin expression and neurogenesis in the hippocampus of adult mice. J. Neurochem. 80, 539-547. doi: 10.1046/j.0022-3042.2001.00747.x

Lin, A. L., Coman, D., Jiang, L., Rothman, D. L., and Hyder, F. (2014). Caloric restriction impedes age-related decline of mitochondrial function and neuronal activity. J. Cereb. Blood Flow Metab. 34, 1440-1443. doi: 10.1038/jcbfm. 2014.114

Lin, A. L., Gao, J. H., Duong, T. Q., and Fox, P. T. (2010). Functional neuroimaging: a physiological perspective. Front. Neuroenergetics 2:17. doi: 10.3389/fnene. 2010.00017

Lin, A. L., Jahrling, J. B., Zhang, W., Derosa, N., Bakshi, V., Romero, P., et al. (2017a). Rapamycin rescues vascular, metabolic and learning deficits in apolipoprotein E4 transgenic mice with pre-symptomatic Alzheimer's disease. J. Cereb. Blood Flow Metab. 37, 217-226.

Lin, A. L., Laird, A. R., Fox, P. T., and Gao, J. H. (2012). Multimodal MRI neuroimaging biomarkers for cognitive normal adults, amnestic mild cognitive impairment, and Alzheimer's disease. Neurol. Res. Int. 2012:907409. doi: 10.1155/2012/907409

Lin, A. L., Parikh, I., Hoffman, J. D., and Ma, D. (2017b). Neuroimaging biomarkers of caloric restriction on brain metabolic and vascular functions. Curr. Nutr. Rep. 6, 41-48. doi: 10.1007/s13668-017-0187-9

Lin, A. L., Powell, D., Caban-Holt, A., Jicha, G., Robertson, W., Gold, B. T., et al. (2016). ${ }^{1} \mathrm{H}$-MRS metabolites in adults with down syndrome: effects of dementia. Neuroimage Clin. 11, 728-735. doi: 10.1016/j.nicl.2016.06.001

Lin, A. L., and Rothman, D. L. (2014). What have novel imaging techniques revealed about metabolism in the aging brain? Future Neurol. 9, 341-354. doi: $10.2217 /$ fnl.14.13

Lin, A. L., Zhang, W., Gao, X., and Watts, L. (2015). Caloric restriction increases ketone bodies metabolism and preserves blood flow in aging brain. Neurobiol. Aging 36, 2296-2303. doi: 10.1016/j.neurobiolaging.2015.03.012

Lin, A. L., Zheng, W., Halloran, J. J., Burbank, R. R., Hussong, S. A., Hart, M. J., et al. (2013). Chronic rapamycin restores brain vascular integrity and function through NO synthase activation and improves memory in symptomatic mice modeling Alzheimer's disease. J. Cereb. Blood Flow Metab. 33, 1412-1421. doi: $10.1038 /$ jcbfm.2013.82

Liu, C. C., Kanekiyo, T., Xu, H., and Bu, G. (2013). Apolipoprotein E and Alzheimer disease: risk, mechanisms and therapy. Nat. Rev. Neurol. 9, 106-118. doi: 10.1038/nrneurol.2012.263

Liu, Y. C., Gao, X. X., Chen, L., and You, X. Q. (2017). Rapamycin suppresses Abeta25-35- or LPS-induced neuronal inflammation via modulation of NFkappaB signaling. Neuroscience 355, 188-199. doi: 10.1016/j.neuroscience.2017. 05.005
Ma, D., Wang, A., Parikh, I., Green, S. J., Hoffman, J. D., Chlipala, G., et al. (2018). Ketogenic diet enhances neurovascular function with altered gut microbiome in young healthy mice. Sci. Rep. 8:6670. doi: 10.1038/s41598-018-25190-5

Ma, D. J., Galanis, E., Anderson, S. K., Schiff, D., Kaufmann, T. J., Peller, P. J., et al. (2015). A phase II trial of everolimus, temozolomide, and radiotherapy in patients with newly diagnosed glioblastoma: NCCTG N057K. Neuro Oncol. 17, 1261-1269. doi: 10.1093/neuonc/nou328

Majumder, S., Richardson, A., Strong, R., and Oddo, S. (2011). Inducing autophagy by rapamycin before, but not after, the formation of plaques and tangles ameliorates cognitive deficits. PLoS One 6:e25416. doi: 10.1371/journal.pone. 0025416

Mannick, J. B., Del Giudice, G., Lattanzi, M., Valiante, N. M., Praestgaard, J., Huang, B., et al. (2014). mTOR inhibition improves immune function in the elderly. Sci. Transl. Med. 6:268ra179. doi: 10.1126/scitranslmed.3009892

Mattson, M. P. (2010). The impact of dietary energy intake on cognitive aging. Front. Aging Neurosci. 2:5. doi: 10.3389/neuro.24.005.2010

McCay, C. M., Crowell, M. F., and Maynard, L. A. (1989). The effect of retarded growth upon the length of life span and upon the ultimate body size. 1935. Nutrition 5, 155-171; discussion 172.

McDaniel, S. S., Rensing, N. R., Thio, L. L., Yamada, K. A., and Wong, M. (2011). The ketogenic diet inhibits the mammalian target of rapamycin (mTOR) pathway. Epilepsia 52, e7-e11. doi: 10.1111/j.1528-1167.2011.02981.x

Mita, M. M., Mita, A., and Rowinsky, E. K. (2003). The molecular target of rapamycin (mTOR) as a therapeutic target against cancer. Cancer Biol. Ther. 2, S169-S177. doi: 10.4161/cbt.365

Moraal, B., Van Den Elskamp, I. J., Knol, D. L., Uitdehaag, B. M., Geurts, J. J., Vrenken, H., et al. (2010). Long-interval T2-weighted subtraction magnetic resonance imaging: a powerful new outcome measure in multiple sclerosis trials. Ann. Neurol. 67, 667-675. doi: 10.1002/ana.21958

Mouton, P. R., Chachich, M. E., Quigley, C., Spangler, E., and Ingram, D. K. (2009). Caloric restriction attenuates amyloid deposition in middle-aged dtg APP/PS1 mice. Neurosci. Lett. 464, 184-187. doi: 10.1016/j.neulet.2009.08.038

Pan, J. W., De Graaf, R. A., Petersen, K. F., Shulman, G. I., Hetherington, H. P., and Rothman, D. L. (2002). [2,4-13 $\left.\mathrm{C}_{2}\right]$-beta-Hydroxybutyrate metabolism in human brain. J. Cereb. Blood Flow Metab. 22, 890-898. doi: 10.1097/00004647200207000-00014

Parikh, I., Guo, J., Chuang, K. H., Zhong, Y., Rempe, R. G., Hoffman, J. D., et al. (2016). Caloric restriction preserves memory and reduces anxiety of aging mice with early enhancement of neurovascular functions. Aging 8, 2814-2826. doi: 10.18632/aging.101094

Park, H. W. (2010). Longevity, aging, and caloric restriction: Clive Maine McCay and the construction of a multidisciplinary research program. Hist. Stud. Nat. Sci. 40, 79-124. doi: 10.1525/hsns.2010.40.1.79

Park, S. Y., Choi, G. H., Choi, H. I., Ryu, J., Jung, C. Y., and Lee, W. (2005). Calorie restriction improves whole-body glucose disposal and insulin resistance in association with the increased adipocyte-specific GLUT4 expression in Otsuka Long-Evans Tokushima fatty rats. Arch. Biochem. Biophys. 436, 276-284. doi: 10.1016/j.abb.2005.01.010

Perluigi, M., Di Domenico, F., and Butterfield, D. A. (2015). mTOR signaling in aging and neurodegeneration: at the crossroad between metabolism dysfunction and impairment of autophagy. Neurobiol. Dis. 84, 39-49. doi: 10.1016/j.nbd.2015.03.014

Rahat, O., Maoz, N., and Cohen, H. Y. (2011). Multiple pathways regulating the calorie restriction response in yeast. J. Gerontol. A Biol. Sci. Med. Sci. 66, 163-169. doi: 10.1093/gerona/glq165

Redman, L. M., Smith, S. R., Burton, J. H., Martin, C. K., Il'yasova, D., and Ravussin, E. (2018). Metabolic slowing and reduced oxidative damage with sustained caloric restriction support the rate of living and oxidative damage theories of aging. Cell Metab. 27, 805.e4-815.e4. doi: 10.1016/j.cmet.2018. 02.019

Reiman, E. M., Caselli, R. J., Chen, K., Alexander, G. E., Bandy, D., and Frost, J. (2001). Declining brain activity in cognitively normal apolipoprotein E epsilon 4 heterozygotes: a foundation for using positron emission tomography to efficiently test treatments to prevent Alzheimer's disease. Proc. Natl. Acad. Sci. U.S.A. 98, 3334-3339. doi: 10.1073/pnas.061509598

Reiman, E. M., Chen, K., Alexander, G. E., Caselli, R. J., Bandy, D., Osborne, D., et al. (2004). Functional brain abnormalities in young adults at genetic risk 
for late-onset Alzheimer's dementia. Proc. Natl. Acad. Sci. U.S.A. 101, 284-289. doi: $10.1073 /$ pnas. 2635903100

Reiman, E. M., Chen, K., Alexander, G. E., Caselli, R. J., Bandy, D., Osborne, D., et al. (2005). Correlations between apolipoprotein E epsilon4 gene dose and brain-imaging measurements of regional hypometabolism. Proc. Natl. Acad. Sci. U.S.A. 102, 8299-8302. doi: 10.1073/pnas.0500579102

Richardson, A., Galvan, V., Lin, A. L., and Oddo, S. (2015). How longevity research can lead to therapies for Alzheimer's disease: the rapamycin story. Exp. Gerontol. 68, 51-58. doi: 10.1016/j.exger.2014.12.002

Roy, M., Hennebelle, M., St-Pierre, V., Courchesne-Loyer, A., Fortier, M., BouzierSore, A. K., et al. (2013). Long-term calorie restriction has minimal impact on brain metabolite and fatty acid profiles in aged rats on a Western-style diet. Neurochem. Int. 63, 450-457. doi: 10.1016/j.neuint.2013.08.006

Sabatini, D. M., Erdjument-Bromage, H., Lui, M., Tempst, P., and Snyder, S. H. (1994). RAFT1: a mammalian protein that binds to FKBP12 in a rapamycin-dependent fashion and is homologous to yeast TORs. Cell 78, 35-43. doi: 10.1016/0092-8674(94)90570-3

Salt, I. P., Johnson, G., Ashcroft, S. J., and Hardie, D. G. (1998). AMP-activated protein kinase is activated by low glucose in cell lines derived from pancreatic beta cells, and may regulate insulin release. Biochem. J. 335(Pt 3), 533-539. doi: 10.1042/bj3350533

Sasongko, T. H., Ismail, N. F., and Zabidi-Hussin, Z. (2016). Rapamycin and rapalogs for tuberous sclerosis complex. Cochrane Database Syst. Rev. 7:CD011272. doi: 10.1002/14651858.CD011272.pub2

Schafer, M. J., Alldred, M. J., Lee, S. H., Calhoun, M. E., Petkova, E., Mathews, P. M., et al. (2015). Reduction of beta-amyloid and gamma-secretase by calorie restriction in female Tg2576 mice. Neurobiol. Aging 36, 1293-1302. doi: 10.1016/j.neurobiolaging.2014.10.043

Sehgal, S. N., Baker, H., and Vezina, C. (1975). Rapamycin (AY-22,989), a new antifungal antibiotic. II. Fermentation, isolation and characterization. J. Antibiot. 28, 727-732. doi: 10.7164/antibiotics.28.727

Serkova, N., Litt, L., James, T. L., Sadee, W., Leibfritz, D., Benet, L. Z., et al. (1999). Evaluation of individual and combined neurotoxicity of the immunosuppressants cyclosporine and sirolimus by in vitro multinuclear NMR spectroscopy. J. Pharmacol. Exp. Ther. 289, 800-806.

Soefje, S. A., Karnad, A., and Brenner, A. J. (2011). Common toxicities of mammalian target of rapamycin inhibitors. Target Oncol. 6, 125-129. doi: 10.1007/s11523-011-0174-9

Spilman, P., Podlutskaya, N., Hart, M. J., Debnath, J., Gorostiza, O., Bredesen, D., et al. (2010). Inhibition of mTOR by rapamycin abolishes cognitive deficits and reduces amyloid-beta levels in a mouse model of Alzheimer's disease. PLoS One 5:e9979. doi: 10.1371/journal.pone.0009979

Stanfel, M. N., Shamieh, L. S., Kaeberlein, M., and Kennedy, B. K. (2009). The TOR pathway comes of age. Biochim. Biophys. Acta 1790, 1067-1074. doi: 10.1016/j.bbagen.2009.06.007

Subbiah, V., Berry, J., Roxas, M., Guha-Thakurta, N., Subbiah, I. M., Ali, S. M., et al. (2015). Systemic and CNS activity of the RET inhibitor vandetanib combined with the mTOR inhibitor everolimus in KIF5B-RET re-arranged non-small cell lung cancer with brain metastases. Lung Cancer 89, 76-79. doi: 10.1016/ j.lungcan.2015.04.004
Thambisetty, M., Beason-Held, L., An, Y., Kraut, M. A., and Resnick, S. M. (2010). APOE epsilon 4 genotype and longitudinal changes in cerebral blood flow in normal aging. Arch. Neurol. 67, 93-98. doi: 10.1001/archneurol.2009.913

Thrasivoulou, C., Soubeyre, V., Ridha, H., Giuliani, D., Giaroni, C., Michael, G. J., et al. (2006). Reactive oxygen species, dietary restriction and neurotrophic factors in age-related loss of myenteric neurons. Aging Cell 5, 247-257. doi: 10.1111/j.1474-9726.2006.00214.x

Tillema, J. M., Leach, J. L., Krueger, D. A., and Franz, D. N. (2012). Everolimus alters white matter diffusion in tuberous sclerosis complex. Neurology 78, 526-531. doi: 10.1212/WNL.0b013e318247ca8d

Valdez, G., Tapia, J. C., Kang, H., Clemenson, G. D. Jr., Gage, F. H., Lichtman, J. W., et al. (2010). Attenuation of age-related changes in mouse neuromuscular synapses by caloric restriction and exercise. Proc. Natl. Acad. Sci. U.S.A. 107, 14863-14868. doi: 10.1073/pnas.1002220107

Van der Auwera, I., Wera, S., Van Leuven, F., and Henderson, S. T. (2005). A ketogenic diet reduces amyloid beta 40 and 42 in a mouse model of Alzheimer's disease. Nutr. Metab. 2:28.

Vanitallie, T. B., Nonas, C., Di Rocco, A., Boyar, K., Hyams, K., and Heymsfield, S. B. (2005). Treatment of Parkinson disease with diet-induced hyperketonemia: a feasibility study. Neurology 64, 728-730. doi: 10.1212/01.WNL.0000152046. 11390.45

Walczyk, T., and Wick, J. Y. (2017). The ketogenic diet: making a comeback. Consult. Pharm. 32, 388-396. doi: 10.4140/TCP.n.2017.388

Wang, Z. J., Bergqvist, C., Hunter, J. V., Jin, D., Wang, D. J., Wehrli, S., et al. (2003). In vivo measurement of brain metabolites using two-dimensional doublequantum MR spectroscopy-exploration of GABA levels in a ketogenic diet. Magn. Reson. Med. 49, 615-619. doi: 10.1002/mrm.10429

Witte, A. V., Fobker, M., Gellner, R., Knecht, S., and Floel, A. (2009). Caloric restriction improves memory in elderly humans. Proc. Natl. Acad. Sci. U.S.A. 106, 1255-1260. doi: 10.1073/pnas.0808587106

Witte, A. V., Kerti, L., Margulies, D. S., and Floel, A. (2014). Effects of resveratrol on memory performance, hippocampal functional connectivity, and glucose metabolism in healthy older adults. J. Neurosci. 34, 7862-7870. doi: 10.1523/ JNEUROSCI.0385-14.2014

Yang, Q., Guo, M., Wang, X., Zhao, Y., Zhao, Q., Ding, H., et al. (2017). Ischemic preconditioning with a ketogenic diet improves brain ischemic tolerance through increased extracellular adenosine levels and hypoxia-inducible factors. Brain Res. 1667, 11-18. doi: 10.1016/j.brainres.2017.04.010

Conflict of Interest Statement: The authors declare that the research was conducted in the absence of any commercial or financial relationships that could be construed as a potential conflict of interest.

Copyright (c) 2018 Lee, Yanckello, Ma, Hoffman, Parikh, Thalman, Bauer, Hartz, Hyder and Lin. This is an open-access article distributed under the terms of the Creative Commons Attribution License (CC BY). The use, distribution or reproduction in other forums is permitted, provided the original author $(s)$ and the copyright owner(s) are credited and that the original publication in this journal is cited, in accordance with accepted academic practice. No use, distribution or reproduction is permitted which does not comply with these terms. 